\title{
ELECCIONES EN GRECIA
}

Bajo el signo del «allaghi», del «cambio», se celebraron el 18 de octubre pasado las elecciones al Parlamento griego que dieron el triunfo al Movimiento Socialista Panhelénico (Pasok). Los resultados finales arrojan 170 escaños para el Pasok, 112 para la Nueva Democracia y 12 para el Partido Comunista del Exterior (Kke), que no logró alcanzar el fatídico 17 por 100 necesario para poder tomar parte en la segunda ronda de distribución de escaños. También el Partido Comunista del Interior (eurocomunista) fue víctima del sistema de representación reforzada, quedando excluido del Parlamento, mientras que las diversas formaciones de extrema izquierda se han convertido en marginales. Tres diputados que se presentaron por la Nueva Democracia (Kanelopoulos, Theotokis y Kokkebis), otros dos elegidos por los votantes del Pasok (Mavros y Glezos) y un diputado comunista han pasado a formar como independientes el grupo mixto (véase cuadro 1.1). En las simultáneas elecciones para designar los veinticuatro representantes griegos en el Parlamento europeo, junto a las tres fuerzas mayoritarias obtuvieron respectivamente un escaño los eurocomunistas, el partido progresista, el partido de la Unión Democrática y la Unión de Centro Democrático, que queda reducido después de haber sido la segunda fuerza política en 1974 a una representación puramente testimonial (véase cuadro 1.2). El triunfo del Pasok puede calificarse de histórico, por cuanto que supone la primera experiencia de gobierno de la izquierda en Grecia desde su creación como Estado independiente en 1830 (cuadro 2).

Aproximadamente 6.800 .000 votantes habían sido convocados a las urnas instaladas en las 18.000 circunscripciones locales para elegir entre 26 partidos y más de 2.500 candidatos a 288 de los 300 miembros que integran el Parlamento unicameral. La designación de los doce parlamentarios restantes - los «diputados de Estado»- correspondía según la ley electoral a los partidos mayoritarios, sobre la base de las listas previamente presentadas por los partidos, en las que figuraban personalidades simpatizantes con su línea política. Aunque la obligatoriedad del voto, que se halla prescrita por la ley, resulta paliada por el hecho de que las sanciones teóricamente imponibles (desde la pérdida del permiso de conducir o de una licencia comercial hasta la retirada del pasaporte) no se aplican generalmente, la abstención alcanzó en este caso la considerable cifra del 21,5 por 100. La ley electoral prevee asimismo que los ciudadanos griegos residentes fuera del país -varios millones de personas, lo que supone un espectro ciudadano de indudable peso en 
relación con los nueve millones y medio de griegos que viven en el país- no tengan derecho al voto.

El sistema electoral griego, que se califica de representación proporcional reforzada, integra elementos del sistema mayoritario existente en algunos países europeos y del de representación simple. Sus efectos son: primar notablemente al primer partido que obtiene relativamente mayor porcentaje de escaños que de votos, favorecer algo menos al segundo y limitar considerablemente la representación del partido o partidos minoritarios restantes. Este complejo sistema de distribución de escaños, que se realiza en tres rondas sucesivas, había jugado claramente a favor de Nueva Democracia en las elecciones de 1974 y 1977. Fundado en 1974 por Constantino Karamanlis a su vuelta al país tras la caída de la dictadura de los coroneles (19671974), Nueva Democracia fue una solución nacional en el tránsito de la dictadura a la democracia parlamentaria. Los griegos acudieron en masa - 54,3 por 100- a esta formación política, fuertemente influidos por el carácter carismático de Karamanlis. Cuatro años más tarde -noviembre de 1977- Nueva Democracia conquistaba de nuevo la mayoría absoluta con el 41,8 por 100 de los votos frente al 25,3 por 100 del Pasok. De cara a las últimas elecciones, el partido en el poder apoyó su campaña electoral en el pragmatismo de sus realizaciones políticas: el tránsito ordenado de la dictadura a la democracia, la cimentación de un nuevo orden político y el desarrollo económico, así como el aumento de la renta per cápita registrados en la nación a pesar de la crisis energética mundial, de la que Grecia se resintió gravemente. Nueva Democracia se presentaba como un partido de centro-derecha, declarándose partidario de la presencia griega en la Alianza Atlántica, a cuyo mando militar Grecia se reintegró a finales de 1980, y del continuismo en la política respecto del Mercado Común, iniciada con el ingreso en enero de 1981. El programa del partido subraya la creencia de sus afiliados en la democracia pluralista y en la libre iniciativa privada, sin omitit la participación del Estado en determinados sectores económicos, así como el expreso rechazo de cualquier régimen de tipo totalitario. En política exterior, Nueva Democracia proclama como objetivos básicos la independencia nacional y la integridad territorial, la coexistencia pacífica y la pertenencia de Grecia al mundo occidental, con el consecuente afianzamiento de sus vínculos políticos, económicos y de defensa. A la cabeza del partido se hallaba el hasta ahora primer ministro, Georgios Rallis, quien sustituyó a Karamanlis tras el pase de éste a la Presidencia de la República en 1980. Rallis enunció durante la campaña diez puntos prioritarios caso de ganar las elecciones. El primero era la reforma de la Administración del Estado, un viejo problema de la estructura política griega, en el que se unen el férreo centralismo administrativo dominante en el país desde su independencia y un lento burocratismo oficial, especialmente favorecedor del clientelismo y de la corrupción. Frente a los problemas derivados de la inflación -el 24 por 100 en los últimos tres años- Nueva Democracia abogaba por el incremento de incentivos a las inversiones privadas, reforma fiscal e impositiva, y combatir la evasión tributaria. ¿Qué ocurrirá en Nueva Democracia tras el fracaso electoral? Rallis será posiblemente sustituido; quizá por el propio Evangelos Averoff, viceprimer ministro en el anterior Gobierno y ministro de Defensa, partidario de una línea ideológica más derechista, aunque no se descarta un líder de nueva imagen, más joven, que no pertenezca a la generación precedente. Un proceso de disgregación entre sus distintos componentes es más que previsible.

Por su parte, el Pasok, fundado en 1974 por Andreas Papandreu, jugaba dos bazas fundamentales: nacionalismo y modernización. De los modestos doce escaños obtenidos en la primera legislatura, el Pasok, único partido socialista europeo no integrado en la Internacional Socialista, paso a ocupar 93 escaños en 1977. El programa de los socialistas antes de los comicios comprendía los siguientes objetivos 
principales: descentralización administrativa, nacionalización y autogestión de un amplio sector productivo (banca, seguros, transportes, industria del cemento y de la construcción naval, minería, acero, industria química), adecuación de los salarios con un mecanismo autónomo ligado al aumento de los precios, amplio movimiento cooperativo agrícola e industrial, estabilización y, posteriormente, reducción de la inflación, nacionalización de los terrenos agrícolas propiedad de la Iglesia ortodoxa, creación de sindicatos libres, estatalización del segundo canal de la televisión nacional, hoy propiedad del Ejército, reforma del sistema educativo, socialización de la Medicina, creación de un seguro nacional de enfermedad, protección del medio ambiente y participación popular en el desarrollo cultural. Con estas medidas, los socialistas preveen un plan de inversiones públicas que debería incrementar el crecimiento económico en un 5 por 100 en los cuatro años de la legislatura. El programa del Pasok incluye además la separación entre la Iglesia y el Estado, la destrucción de los archivos políticos, que se remontan a la guerra civil griega, la igualdad entre hombres y mujeres, y la participación democrática de los ciudadanos en el desarrollo de la vida política del país.

Se considera unánimemente como más conflictiva la política exterior de Papandreu. Se autocalifica de independiente y presenta entre sus principales metas la garantía de la integridad territorial de Grecia, la eliminación de las armas nucleares en el área de los Balcanes y la neutralización del Mediterráneo. El Pasok afirma que Grecia debe retirarse de las alianzas militares, políticas y económicas, que «minan la independencia nacional», y afirma el derecho del pueblo griego a dirigir por sí mismo el curso del país. Él socialismo nacionalista de Papandreu orientó su campaña electoral sobre la clave de una política nueva e intransigente en relación con los dos contenciosos grecoturcos abiertos en la actualidad: el virtual dominio turco sobre Chipre (cuya población es en un 85 por 100 greco-chipriota) y la disputa sobre la plataforma continental del mar Egeo, rica en petróleo. La misma afirmación programática del Pasok sobre la salida griega de la Alianza Atlántica tiene como motivación el trato preferente que la OTAN concede a Turquía - 34 bases americanas en Turquía, sólo cuatro en Grecia- y la tácita aceptación por la Alianza Atlántica de la invasión turca de Chipre en 1974, invasión que se produjo en apoyo de una minoría turca que representa únicamente un 18 por 100 de la población de la isla. Así pues, una política exterior claramente nacionalista y de un neto rechazo tanto de la OTAN como de la CEE, lo que lógicamente produjo serias inquietudes en Washington y Bruselas tras el triunfo socialista. Ello explica que el secretario norteamericano de Defensa, Caspar Weinberger, se mostrase «preocupado» y deseo de que Grecia no abandone la Alianza durante la reunión que el comité de planes nucleares de la OTAN inició en Escocia poco después de que se conocieran los resultados de las elecciones griegas. La preocupación en Washington se entiende también desde la óptica de la amenaza que el triunfo del Pasok supone para el mantenimiento de la relación bilateral en materia de defensa con los Estados Unidos, que, como en el caso de España, se encuentra en suspenso desde que el pasado mes de junio, el propio Gobierno proamericano de Rallis abandonara la negociación por falta de contrapartidas.

¿Qué posibilidades reales tiene Papandreu de realizar su programa? El líder socialista anunció la noche de su triunfo que los «ciudadanos de este país comprobarán en el plazo de pocos días la realidad de los cambios que haremos». El programa gubernamental, presentado el domingo 22 de noviembre ante el Parlamento, reflejó fielmente las promesas electorales. En el plano interior, Papandreu subrayó la «socialización» - término expresamente preferido al de «nacionalización»- de todas las empresas de «utilidad pública» o que trabajen para la defensa nacional, junto a las empresas de transportes, comunicaciones, industrias farmacéuticas, ce- 
mentos, aceros y algunos astilleros. El proceso será puesto paulatinamente en marcha y los afectados serán indemnizados por el Estado. Los gastos militares - según el primer ministro todavía superiores en 1982 a los de educación, sanidad o seguridad social - han de permitir a la industria bélica griega desarrollarse para que pueda exportar una parte de su producción. Otras medidas anticipadas por $\mathrm{Pa}$ pandreu: la puesta en marcha de un plan económico quinquenal para frenar la inflación, la anunciada separación total de la Iglesia y el Estado y la adopción del matrimonio civil en tanto que «matrimonio paralelo y jurídicamente equivalente al religioso». En política exterior, destacan la anunciada desnuclearización de los Balcanes, la continuación del diálogo con Ankara sin ningún tipo de concesiones al Gobierno turco, un calendario de retirada de las bases norteamericanas en Grecia y el efectivo control operacional del mar Egeo, lo que supone la disolución del acuerdo Rogers, por el cual se permitía a Grecia reintegrarse al mando militar de la OTAN. Por lo que respecta al Mercado Común, Papandreu inauguró el 26 de noviembre la «cumbre» de la CEE en Londres exigiendo un estatuto especial para su país que salvaguarde los intereses económicos griegos, y aludió a la posibilidad de convocar un referéndum para decidir sobre la salida o permanencia griega en la Comunidad Económica Europea.

El referéndum tendría que salvar, sin embargo, una serie de escollos. La posibilidad de concederlo depende de Karamanlis, que fue precisamente el artífice de la adhesión. La propuesta de referéndum debería obtener en segundo lugar el apoyo de los dos tercios de los diputados de un Parlamento donde socialistas y comunistas suman 182 escaños, que son 17 menos de los necesarios para llegar a la mayoría cualificada. Además, Papandreu podría perder la consulta popular incluso en la calle. Un 10 por 100 de los que votaron Pasok para el Parlamento griego eligieron diputados de otras tendencias para el Parlamento europeo. Finalmente, hay que tener en cuenta la fuerte presión de los militares, unánimemente otanistas y partidarios de la integración europea, sin descartar las ofertas exteriores, que podrían suponer una contrapartida aceptable para los socialistas.

Papandreu ha asumido paralelamente a la presidencia del Consejo el Ministerio de Defensa. Para comprender el latente peligro de golpe militar y la presión política que ello supone hay que descender a una visión sumaria de las fuerzas armadas griegas. Los altos mandos son leales al régimen y no es previsible una actitud de interferencia en el poder civil. Entre los jefes y mandos intermedios es donde permanece una actitud nostálgica hacia el pasado de la dictadura militar. La clase de tropa participa del clima popular favorable al Pasok. La política prometida por el Gobierno de refuerzo y modernización de las fuerzas armadas constriñe a los socialistas por otra parte a permanecer en la Alianza Atlántica.

En definitiva, las elecciones griegas hacen patentes una serie de elementos para el análisis político de la estricta evolución interna en Grecia, pero también de su posible repercusión y analogía con otros países. Entre ellos, cabe señalar: la gran dinámica de la "voluntad de cambio», expresada ya nítidamente en las elecciones francesas; el progresivo desgaste del centro político; el retorno de la atractividad de opciones ideológicas claras; el resurgimiento de los nacionalismos - detectable en toda Europa- y la apropiación de su potencial político por la izquierda, junto a un afianzamiento de los regionalismos localistas y del deseo de participación real en el poder; por último, la indudable efectividad política de los movimientos pacifistas, sin olvidar lo que la actitud del actual Gobierno griego frente a la OTAN supone de revalorización de la opción atlántica en el caso concreto de España.

José M.a Beneyto PÉrez 


\section{Cuadro 1.1}

\section{ELECCIONES AL PARLAMENTO GRIEGO}

\begin{tabular}{|c|c|c|c|c|c|c|c|c|c|}
\hline$\frac{\text { Partidos }}{\text { R. electorales }}$ & $\begin{array}{c}\text { Nueva } \\
\text { Democracia }\end{array}$ & $\begin{array}{c}\text { Pasok } \\
\text { (socialistas) }\end{array}$ & $\begin{array}{l}\text { Unión de } \\
\text { Centro } \\
\text { Democrático } \\
\text { (EDIK) }\end{array}$ & $\begin{array}{c}\text { Partido } \\
\text { Comunista } \\
\text { del Interior } \\
\text { (KKE- } \\
\text { ESOT.) * }\end{array}$ & $\begin{array}{l}\text { Partido } \\
\text { Comunista } \\
\text { del Exterior } \\
\text { (KKE) }\end{array}$ & $\begin{array}{c}\text { Frente } \\
\text { Nacional } \\
\text { (extrema } \\
\text { derecha) }\end{array}$ & $\begin{array}{c}\text { Partido } \\
\text { Neo-Liberal }\end{array}$ & $\begin{array}{c}\text { Unión } \\
\text { Nacional } \\
\text { Democrática }\end{array}$ & $\begin{array}{c}\text { Abstención } \\
(\%)\end{array}$ \\
\hline \multicolumn{10}{|l|}{ 1974: } \\
\hline Votos ...... & 2.669 .133 & 666.413 & 1.002 .559 & - & 464.787 & - & - & 52.768 & \\
\hline$\% \ldots \ldots \ldots$ & 54,37 & 13,58 & 20,42 & - & 9,47 & - & - & 1,08 & 20,47 \\
\hline Escaños $\ldots$ & 220 & 12 & 60 & - & 8 & - & 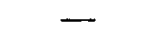 & - & \\
\hline \multicolumn{10}{|l|}{ 1977: } \\
\hline Votos ... ... & 2.146 .365 & 1.300 .025 & 612.786 & 139.356 & 480.272 & 349.988 & 55.498 & 一 & \\
\hline$\% \ldots \ldots \ldots$ & 41,84 & 25,34 & 11,95 & 2,72 & 9,36 & 6,82 & 1,08 & - & 20,1 \\
\hline Escaños $\quad .$. & 171 & 93 & 16 & 2 & 11 & 5 & 2 & - & \\
\hline \multicolumn{10}{|l|}{ 1981: } \\
\hline Votos ...... & 2.033 .584 & 2.725 .132 & 23.723 & 77.461 & 619.292 & - & - & - & \\
\hline$\% \ldots \ldots \ldots$ & 35,86 & 48,06 & 0,42 & 1,37 & 10,92 & - & 一 & - & 21,5 \\
\hline Escaños $\quad .$. & 112 & 170 & - & - & 12 & - & - & - & \\
\hline
\end{tabular}

* El Partido Comunista del Interior concurrió como Alianza de Izquierda en 1974 junto al KKE y en 1977 con otros partidos de izquierda. Fuente: Oficina de Prensa. Embajada de Grecia. 


\section{Cuadro 1.2}

\section{ELECCIONES AL PARLAMENTO EUROPEO}

\begin{tabular}{|c|c|c|c|c|c|c|c|}
\hline $\begin{array}{c}\text { Partidos } \\
\text { Resultados }\end{array}$ & Pasok & $\begin{array}{c}\text { Nueva } \\
\text { Democracia }\end{array}$ & $\begin{array}{c}\text { Partido } \\
\text { Comunista } \\
\text { del Exterior }\end{array}$ & $\begin{array}{c}\text { Partido } \\
\text { Comunista } \\
\text { del Interlor }\end{array}$ & $\begin{array}{c}\text { Unión } \\
\text { de Centro } \\
\text { Democrático }\end{array}$ & $\begin{array}{c}\text { Partido } \\
\text { Progresista }\end{array}$ & $\begin{array}{c}\text { Partido de } \\
\text { la Unión } \\
\text { Democrática }\end{array}$ \\
\hline $\begin{array}{llll}\text { Votos } & \ldots & \ldots \\
\% & \ldots & \ldots & \ldots \\
\text { Escaños } & \ldots & \ldots\end{array}$ & $\begin{array}{c}2.155 .434 \\
40,29 \\
10\end{array}$ & $\begin{array}{c}1.686 .876 \\
31,53 \\
8\end{array}$ & $\begin{array}{c}678.236 \\
12,68 \\
3\end{array}$ & $\begin{array}{c}275.731 \\
5,15 \\
1\end{array}$ & $\begin{array}{c}60.843 \\
1,14 \\
1\end{array}$ & $\begin{array}{c}104.551 \\
1,95 \\
1\end{array}$ & $\begin{array}{c}223.072 \\
4,17 \\
1\end{array}$ \\
\hline
\end{tabular}




\section{CuAdro 2}

EVOLUCION DEL VOTO EN GRECIA DESDE 1946 HASTA LA DICTADURA DE LOS CORONELES (1967) *

\begin{tabular}{|c|c|c|c|c|c|c|c|c|c|}
\hline & 1946 & 1950 & 1951 & 1952 & 1956 & 1958 & 1961 & 1963 & 1964 \\
\hline $\begin{array}{l}\text { Unión Radical } \\
\text { Nacional ** }\end{array}$ & $\begin{array}{c}610.935 \\
55,12 \\
206\end{array}$ & $\begin{array}{c}317.512 \\
18,80 \\
62\end{array}$ & $\begin{array}{c}624.316 \\
36,56 \\
114\end{array}$ & $\begin{array}{c}783.541 \\
49,22 \\
240\end{array}$ & $\begin{array}{c}1.594 .112 \\
47,88 \\
165\end{array}$ & $\begin{array}{c}1.583 .885 \\
41,16 \\
171\end{array}$ & $\begin{array}{c}2.347 .824 \\
50,80 \\
176\end{array}$ & $\begin{array}{c}1.837 .377 \\
39,37 \\
132\end{array}$ & $\begin{array}{c}1.621 .546 \\
35,26 \\
107\end{array}$ \\
\hline $\begin{array}{l}\text { Unión } \\
\text { del Centro *** }\end{array}$ & $\begin{array}{c}213.721 \\
19,28 \\
68\end{array}$ & $\begin{array}{c}277.739 \\
16,44 \\
45\end{array}$ & $\begin{array}{c}401.397 \\
23,49 \\
74\end{array}$ & $\begin{array}{c}544.834 \\
34,22 \\
57\end{array}$ & $\begin{array}{c}1.620 .007 \\
48,15 \\
132\end{array}$ & & $\begin{array}{c}1.555 .442 \\
33,65 \\
100\end{array}$ & $\begin{array}{c}1.962 .079 \\
42,04 \\
138\end{array}$ & $\begin{array}{c}2.424 .477 \\
52,71 \\
171\end{array}$ \\
\hline Partido Liberal & $\begin{array}{c}159.525 \\
14,39 \\
48\end{array}$ & $\begin{array}{c}291.083 \\
17,24 \\
56\end{array}$ & $\begin{array}{c}325.390 \\
19,04 \\
57\end{array}$ & & & $\begin{array}{c}795.445 \\
20,67 \\
36\end{array}$ & & & \\
\hline $\begin{array}{l}\text { Unión de Izquierda } \\
\text { Democrática }\end{array}$ & & $\begin{array}{c}163.824 \\
9,70 \\
18\end{array}$ & $\begin{array}{c}180.640 \\
10,57 \\
10\end{array}$ & $\begin{array}{c}152: 001 \\
9,54 \\
-\end{array}$ & & $\begin{array}{c}939.902 \\
24,42 \\
79\end{array}$ & & $\begin{array}{c}669.267 \\
14,34 \\
28\end{array}$ & $\begin{array}{c}542.865 \\
11,80 \\
22\end{array}$ \\
\hline Partido Agrario & $\begin{array}{c}7.447 \\
0,087 \\
1\end{array}$ & & & & & $\begin{array}{c}468.787 \\
10,62 \\
10\end{array}$ & $\begin{array}{c}675.867 \\
14,62 \\
24\end{array}$ & & \\
\hline
\end{tabular}

* Se recogen únicamente los resultados respectivos de las fuerzas políticas más representativas. Frente de Unidad Nacional, Partido Popular o Rally Griego. i.......

*** Bajo la Unión del Centro como fuerza política de centro-izquierda se recogen en el cuadro los resultados correspondientes en 1946 a la Unión Política Nacional y en 1956 a la Unión Democrática.

Fuente: Oficina de Prensa. Embajada de Grecia. 